\title{
MEASURE ALGEBRAS AND FUNCTIONS OF BOUNDED VARIATION ON IDEMPOTENT SEMIGROUPS( $\left.{ }^{1}\right)$
}

\author{
BY
}

\author{
STEPHEN E. NEWMAN
}

\begin{abstract}
Our main result establishes an isomorphism between all functions on an idempotent semigroup $S$ with identity, under the usual addition and multiplication, and all finitely additive measures on a certain Boolean algebra of subsets of $S$, under the usual addition and a convolution type multiplication. Notions of a function of bounded variation on $S$ and its variation norm are defined in such a way that the above isomorphism, restricted to the functions of bounded variation, is an isometry onto the set of all bounded measures. Our notion of a function of bounded variation is equivalent to the classical notion in case $S$ is the unit interval and the "product" of two numbers in $S$ is their maximum.
\end{abstract}

1. Introduction. This paper is motivated by the pair of closely related Banach algebras described below. The interval $[0,1]$ is an idempotent semigroup when endowed with the operation of maximum multiplication $(x \cdot y=\max (x, y)$ for all $x, y$ in $[0,1]$ ). We let $A$ denote the Boolean algebra (or set algebra) consisting of all finite unions of left-open, right-closed intervals (including the single point 0 ) contained in the interval $[0,1]$. The space $M(A)$ of all bounded, finitely additive measures on $A$ is a Banach space with the usual total variation norm. The nature of the set algebra $A$ and the idempotent multiplication given the interval $[0,1]$ make it possible to define a convolution multiplication in $M(A)$ which makes it a Banach algebra. The convolution product $\mu * \nu$ of two measures $\mu$ and $\nu$ in $M(A)$ is defined by

$$
\mu * \nu(E)=\iint \chi_{E}(x \cdot y) d \mu(x) d \nu(y)
$$

for any set $E$ in $A$, where $\chi_{E}$ denotes the characteristic function of the set $E$. This is the standard way to define the convolution product of two bounded, regular Borel measures on a locally compact semigroup, where the set algebra in question is the $\sigma$-algebra of all Borel subsets of the topological semigroup.

Presented to the Society, January 25, 1969 under the title Functions of bounded variation on idempotent semigroups; received by the editors April 8, 1970.

AMS 1970 subject classifications. Primary 43A10.

Key words and phrases. Bounded variation, convolution measure algebra.

${ }^{(1)}$ The results in this paper are included in the author's doctoral dissertation written at the University of Utah under the direction of Professor Joseph L. Taylor, whose prodding, harassing, encouraging remarks are written between the lines.

Copyright (C) 1972, American Mathematical Society 
The space $B V[0,1]$ consisting of all functions of bounded variation on the interval $[0,1]$, normed by $\|f\|=|f(0)|+V(f)$ for $f$ in $B V[0,1](V(f)$ is the total variation of the function $f$ ), is a well-known Banach space. Moreover, $B V[0,1]$ is a Banach algebra with the above norm and with pointwise multiplication of functions as the multiplication. The algebra $B V[0,1]$ and the algebra $M(A)$ are closely related. Each measure $\mu$ in $M(A)$ determines a function $f$ in $B V[0,1]$ by $f(x)=\mu([0, x])$ for each $x$ in $[0,1]$; conversely, each function in $B V[0,1]$ has this form for some measure in $M(A)$. This correspondence is an isomorphism-isometry which takes the convolution product of two measures to the pointwise of the two corresponding functions. Bounded, regular Borel measures on $[0,1]$, when restricted to be measures on the set algebra $A$, correspond to right continuous functions in $B V[0,1]$; continuous Borel measures correspond to continuous functions in $B V[0,1]$ which are zero at $x=0$; and Borel measures absolutely continuous with respect to Lebesgue measure correspond to absolutely continuous functions which are zero at $x=0$. These results will follow from theorems proved in this paper.

The first two paragraphs illustrate how an algebra of bounded, finitely additive measures, with convolution multiplication, can be represented as an algebra of functions of bounded variation, with pointwise multiplication. This type of representation plays the central role in this paper. In $\$ 2$, we consider a commutative, idempotent semigroup $S$ (not necessarily a topological semigroup), and we let $T$ be a semigroup of semicharacters on $S$ containing the identity semicharacter. A semicharacter on a semigroup $S$ is a bounded, nonzero, complex valued function $f$ on $S$ which satisfies $f(x \cdot y)=f(x) f(y)$ for all $x, y$ in $S$. The functions $\chi_{[0, x)}$ and $\chi_{[0, x]}(x \in[0,1])$ are semicharacters on the semigroup $[0,1]$ with maximum multiplication; in this case, $T$ may consist of all such semicharacters or may consist of simply the semicharacters $\chi_{[0, x]}(x \in[0,1])$. We let $A$ denote the Boolean algebra of subsets of $S$ generated by kernels of semicharacters in $T$. In our example, $A$ would be the set algebra of finite unions of left-open, right-closed intervals (including the single point 0$)$ if we choose $T=\left\{\chi_{[0, x]} \mid x \in[0,1]\right\}$. A convolution multiplication can be introduced in the space of all finitely additive (not necessarily bounded) measures on $A$. Each finitely additive measure $\mu$ on $A$ determines a function $\hat{\mu}$ defined on $T$ by $\hat{\mu}(f)=\mu(S \backslash\{x \in S \mid f(x)=0\})$ for each $f$ in $T$; conversely, each function on $T$ has this form for some finitely additive measure on $A$. The correspondence $\mu \rightarrow \hat{\mu}$ is an isomorphism which takes the convolution product of two finitely additive measures to the pointwise product of the two corresponding functions. This result is purely algebraic in nature; it does not involve norms on the algebras in question.

The notion of a function of bounded variation on $T$ is defined in $\S 3$ in analogy with the usual notion of a function of bounded variation on the unit interval. In fact, these notions are equivalent in our example if we choose $T=\left\{\chi_{[0, x]} \mid x \in[0,1]\right\}$ and we identify the semicharacters in $T$ with the corresponding points in $[0,1]$. A norm is introduced in the space $B V(T)$ of all functions of bounded variation on 
$T$ which makes it a Banach algebra with respect to pointwise multiplication and total variation norm. The space $M(A)$ of all bounded, finitely additive measures is also a Banach algebra with respect to convolution multiplication and total variation norm. The isomorphism $\mu \rightarrow \hat{\mu}$ mentioned above carries bounded measures to functions of bounded variation in a norm-preserving fashion. Hence, the Banach algebras $M(A)$ and $B V(T)$ are isomorphic and isometric. The concept of an absolutely continuous function on $[0,1]$ motivates us to define a similar concept relating pairs of functions in $B V(T)$. This concept allows us to discuss a RadonNikodym theorem in our setting, which generalizes the theory of differentiation on $[0,1]$.

We observe in $\S 4$ that the essential properties of the algebra $B V(T)$, such as bounded variation and norm, depend on $T$ being a commutative, idempotent semigroup with identity, but do not depend on $T$ being a semigroup of semicharacters on some underlying semigroup $S$. Accordingly, we let $T$ be a commutative, idempotent semigroup with identity and we show that $B V(T)$ is a semisimple, commutative convolution measure algebra in the sense of [8]. Taylor showed in [8] that for a semisimple, commutative convolution measure algebra $M$, there is a compact topological semigroup $S$, called the structure semigroup of $M$, and an embedding $\mu \rightarrow \mu_{S}$ of $M$ into $M(S)$ such that each complex homomorphism of $M$ has the form $h_{f}(\mu)=\int_{S} f d \mu_{S}$ for some continuous semicharacter $f$ on $S$. Thus the set $\hat{S}$ of all continuous semicharacters on $S$ can be considered the space of all complex homomorphisms of $M$ (the maximal ideal space of $M$ ). Accordingly, we let $S$ be the structure semigroup of the algebra $B V(T)$. We show that the point evaluation maps are complex homomorphisms of $B V(T)$, and, as such, form an idempotent subsemigroup of the maximal ideal space $\hat{S}$ of $B V(T)$. We use results obtained in [5] to exhibit an idempotent semigroup $T$ such that the structure semigroup $S$ of the algebra $B V(T)$ is not idempotent. We conclude that $\hat{S}$ is not idempotent, even though $T$ is embedded in $\hat{S}$ as an idempotent subsemigroup which separates points in $B V(T)$.

2. Relationships between functions and finitely additive measures on idempotent semigroups. Let $S$ be an abelian idempotent semigroup. Let $T$ be a semigroup of semicharacters on $S$ containing the identity semicharacter. A semicharacter on a semigroup $S$ is a nonzero, bounded, complex valued function on $S$ which is a semigroup homomorphism. A semicharacter on an idempotent semigroup is an idempotent function, and hence can assume only the values zero and one. We define $A_{f}=\{s \in S \mid f(s)=1\}$ and $J_{f}=\{s \in S \mid f(s)=0\}$ for each $f \in T$, and we note that $J_{f}$ is a prime ideal in $S$. A prime ideal in a semigroup is an ideal whose complement is a subsemigroup. Let $A$ denote the Boolean algebra of subsets of $S$ generated by the sets $J_{f}(f \in T)$. If $X=\left\{f_{1}, \ldots, f_{n}\right\}$ is a finite subset of $T, \sigma \in T_{n}\left(T_{n}\right.$ denotes the Boolean algebra of all $n$-tuples of zeros and ones), we define

$$
B(X, \sigma)=\left\{\bigcap_{\sigma(i)=1} A_{f_{i}}\right\} \cap\left\{\bigcap_{\sigma(i)=0} J_{f_{i}}\right\}
$$


Sets of the form (1) will he called fundamental sets. Clearly, the set algebra $A$ consists of finite unions of fundamental sets.

The following two lemmas establish "partitioning" properties of the fundamental sets. The proofs of these lemmas are routine and we omit them.

Lemma 2.1. The sets $\{B(X, \sigma)\}$ for $\sigma \in T_{n}$ form a partition of $S$, where $X=$ $\left\{f_{1}, \ldots, f_{n}\right\}$ is a finite subset of $T$.

LemMA 2.2. The partition $\{B(Y, \tau)\}$ is a refinement of the partition $\{B(X, \sigma)\}$, where $X \subset Y$ are finite subsets of $T$.

We call a complex valued function $g$ on $S$ a simple function (more precisely, a simple function with respect to the set algebra $A$ ) if $g$ assumes only a finite number of values and the set $g^{-1}(z) \in A$ for each $z \in C$.

Lemma 2.3. The set of all simple functions on $S$ is exactly $L(T)$, the linear span of $T$.

Proof. Let $g$ be a simple function on $S$ whose range is the set $\left\{a_{1}, \ldots, a_{m}\right\}$. If $H_{i}=g^{-1}\left(a_{i}\right)$, then $H_{i} \in A$ and the collection $\left\{H_{i}\right\}$ is a partition of $S$. As a result of Lemma 2.2, there exists a finite subset $X=\left\{f_{1}, \ldots, f_{n}\right\}$ of $T$ such that $\{B(X, \sigma)\}$ is a refinement of $\left\{H_{i}\right\}$. Choose constants $b_{\sigma}\left(\sigma \in T_{n}\right)$ as follows: let $b_{\sigma}=0$ if $B(X, \sigma)=\varphi$, and let $b_{\sigma}=a_{i}$ if $B(X, \sigma) \neq \varphi$ and $B(X, \sigma) \subset H_{i}$. Clearly, $g=\sum_{\sigma \in T_{n}} b_{\sigma} \chi_{B(X, \sigma)}$. It follows from (1) that

$$
\chi_{B(X, \sigma)}=\left[\prod_{\sigma(i)=1} f_{i}\right]\left[\prod_{\sigma(i)=0}\left(1-f_{i}\right)\right]
$$

is in $L(T)$ for each $\sigma \in T_{n}$. Thus $g \in L(T)$.

Conversely, let $g=\sum_{i=1}^{n} a_{i} f_{i} \in L(T)\left(a_{i} \in C, f_{i} \in T\right)$. If $X=\left\{f_{1}, \ldots, f_{n}\right\}$, then $g$ is constant on the sets $B(X, \sigma)\left(\sigma \in T_{n}\right)$. If we let $b_{\sigma}=0$ if $B(X, \sigma)=\varphi$, and $b_{\sigma}$ equal the value of $g$ on $B(X, \sigma)$ if $B(X, \sigma) \neq \varphi$, then clearly $g=\sum_{\sigma \in T_{n}} b_{\sigma} \chi_{B(X, \sigma)}$. Thus $g$ is a simple function with respect to $A$.

LEMMA 2.4. Let $\left\{f_{i}\right\}_{i=1}^{n}$ be a set of distinct semicharacters in $T$ and let $\left\{a_{i}\right\}_{i=1}^{n}$ be complex numbers. If $\sum_{i=1}^{n} a_{i} f_{i}=0$, then $a_{i}=0$ for $i=1, \ldots, n$.

Proof. We may assume without loss of generality that $f_{i} \$ f_{j}$ for $i<j$. The result is trivial if $n=1$ since a semicharacter is a nonzero function. Suppose $n>1$. For each $k(1<k \leqq n)$, there is an $s_{k}$ in $S$ such that $s_{k} \in A_{f_{1}} \cap J_{f_{k}}$. If this were not the case, then $A_{f_{1}} \cap J_{f_{k}}=\varphi$, and $A_{f_{1}}$ would be a subset of $A_{f_{k}}$. But then $f_{1} \leqq f_{k}$, which is impossible by our assumption. If we now set $s=s_{2} \cdot s_{3} \cdots s_{n}$, and recall that $A_{f_{1}}$ is a subsemigroup and each $J_{f_{k}}$ is an ideal, we conclude that $s=s_{2} \cdot s_{3} \cdots s_{n}$ is in the set $A_{f_{1}} \cap J_{f_{2}} \cap \cdots \cap J_{f_{n}}$. Thus

$$
a_{1}=\sum_{i=1}^{n} a_{i} f_{i}(s)=0,
$$

and hence $a_{1}=0$. Continuing this process by induction yields $a_{i}=0$ for $i=1, \ldots, n$. This establishes the lemma. 
We now introduce a "convolution" type multiplication into the space of all finitely additive measures on $A$. Let $A \times A$ denote the algebra (not $\sigma$-algebra) of subsets of $S \times S$ generated by rectangles $E \times F$, where $E, F \in A$. If $\mu$ and $\nu$ are finitely additive measures on $A$, then $\mu \times \nu$ is that finitely additive measure on $A \times A$ whose value on any rectangle $E \times F$ is $\mu(E) \nu(F)$. A convolution product of $\mu$ and $\nu$ is defined by

$$
\mu * \nu(E)=\mu \times \nu\left(\alpha^{-1}(E)\right) \quad(E \in A),
$$

where $\alpha: S \times S \rightarrow S$ is the semigroup multiplication on $S$. The following lemma shows that $\alpha^{-1}(E) \in A \times A$ for each $E \in A$, and makes (3) meaningful.

LEMMA 2.5. If $\alpha: S \times S \rightarrow S$ is the multiplication map on $S$, then the inverse image $\alpha^{-1}(E)$ of any set $E$ in $A$ is contained in the algebra $A \times A$.

Proof. It suffices to show that a fundamental set in $A$ is contained in the algebra $A \times A$. Accordingly, let $B(X, \sigma)$ be a fundamental set in $A$ where $X=\left\{f_{1}, \ldots, f_{n}\right\}$ is a subset of $T$ and $\sigma$ is in $T_{n}$. We claim that

$$
\alpha^{-1}(B(X, \sigma))=\bigcup_{\tau_{1} \wedge \tau_{2}=\sigma} B\left(X, \tau_{1}\right) \times B\left(X, \tau_{2}\right)
$$

where $\tau_{1} \wedge \tau_{2}(i)=\min \left(\tau_{1}(i), \tau_{2}(i)\right)$ for $1 \leqq i \leqq n$. We first show that if $(s, t) \in B\left(X, \tau_{1}\right)$ $\times B\left(X, \tau_{2}\right)$ and $\tau_{1} \wedge \tau_{2}=\sigma$, then $s \cdot t \in B(X, \sigma)$. If $\tau_{1} \wedge \tau_{2}(i)=\sigma(i)=1$, then $\tau_{1}(i)=$ $\tau_{2}(i)=1$; thus $s \in A_{f_{i}}, t \in A_{f_{i}}$, and hence $s \cdot t \in A_{f_{i}}$ since $A_{f_{i}}$ is a subsemigroup. If $\tau_{1} \wedge \tau_{2}(i)=\sigma(i)=0$, then either $\tau_{1}(i)=0$ or $\tau_{2}(i)=0$; thus at least one of the points $s$ and $t$ is in $J_{f_{i}}$, and hence $s \cdot t \in J_{f_{i}}$ since $J_{f_{i}}$ is an ideal. Therefore,

$$
s \cdot t \in\left[\bigcap_{\sigma(i)=1} A_{f_{i}}\right] \cap\left[\bigcap_{\sigma(i)=0} J_{f} .\right]=B(X, \sigma) .
$$

We next show that if $s \cdot t \in B(X, \sigma)$, then $s \in B\left(X, \tau_{1}\right)$ and $t \in B\left(X, \tau_{2}\right)$ for some pair $\tau_{1}, \tau_{2}$ in $T_{n}$ with $\tau_{1} \wedge \tau_{2}=\sigma$. Observe that since $\{B(X, \sigma)\}_{\sigma \in T_{n}}$ is a partition of $S$, we must have $s \in B\left(X, \tau_{1}\right)$ and $t \in B\left(X, \tau_{2}\right)$ for some pair $\tau_{1}, \tau_{2}$ in $T_{n}$. If $\sigma(i)=1$, then $s \cdot t \in A_{f_{i}}$, and hence both $s$ and $t$ are in $A_{f_{i}}$ since the complement of $A_{f_{i}}$ is an ideal. Thus $\tau_{1}(i)=\tau_{2}(i)=1$, and $\tau_{1} \wedge \tau_{2}(i)=\sigma(i)$. If $\sigma(i)=0$, then $s \cdot t \in J_{f_{i}}$, and hence one of the points $s$ and $t$ is in $J_{f_{i}}$ since $J_{f_{t}}$ is a prime ideal. Therefore, either $\tau_{1}(i)=0$ or $\tau_{2}(i)=0$, and $\tau_{1} \wedge \tau_{2}(i)=\sigma(i)=0$. Thus $\tau_{1} \wedge \tau_{2}=\sigma$. Our claim is established, and the proof of the lemma is complete.

THEOREM 2.1. There is an isomorphism $\mu \rightarrow \hat{\mu}$ between the algebra of all finitely additive measures on $A$, with convolution multiplication, and the algebra of all functions on $T$, with pointwise multiplication. The function $\hat{\mu}$ is defined by $\hat{\mu}(f)=\mu\left(A_{f}\right)$ $=\int_{S} f d \mu$ for each $f$ in $T$.

Proof. We first notice that $\mu \rightarrow \hat{\mu}$ is clearly a linear map. The integral $\int_{S} g d \mu$ of a simple function $g$ with respect to $\mu$ is defined in the usual way, and makes perfectly good sense even when $\mu$ is not a bounded measure. In fact, the map 
$g \rightarrow \int g d \mu$ is a linear functional on the linear space of all simple functions on $S$. This justifies our use of the integral sign in the statement of the theorem.

To show that $\mu \rightarrow \hat{\mu}$ is onto, let $F$ be a function on $T$. We extend $F$ to a linear functional on $L(T)$ by defining $F\left(\sum_{i=1}^{n} a_{i} f_{i}\right)=\sum_{i=1}^{n} a_{i} F\left(f_{i}\right)$ for any linear combination of semicharacters $\sum_{i=1}^{n} a_{i} f_{i}$ in $L(T)$. Lemma 2.4 assures us that $F$ is a well-defined linear functional on $L(T)$. Lemma 2.3 shows that $L(T)$ is exactly the linear span of all simple functions. We may, therefore, consider $F$ to be a linear functional on the space of all simple functions. Define a measure $\mu$ on $A$ by $\mu(E)=F\left(\chi_{E}\right)$ for any set $E$ in $A$. The measure $\mu$ is finitely additive because $F$ is a linear functional on the simple functions. Furthermore, $\hat{\mu}(f)=\mu\left(A_{f}\right)=F\left(\chi_{A_{f}}\right)=F(f)$ for each $f$ in $T$, and hence $\hat{\mu}=F$. Thus $\mu \rightarrow \hat{\mu}$ is onto.

We employ a similar technique to show that $\mu \rightarrow \hat{\mu}$ is one-to-one. The function $\hat{\mu}$ on $T$ can be extended to a linear functional on the space of simple functions in exactly the same way that $F$ was extended in the previous paragraph. As such, $\hat{\mu}(g)=\int g d \mu$ for each simple function $g$. To prove this, write $g=\sum_{i=1}^{n} a_{i} f_{i}$ as a linear combination of semicharacters. We obtain

$$
\begin{aligned}
\int g d \mu & =\int \sum_{i=1}^{n} a_{i} f_{i} d \mu=\sum_{i=1}^{n} a_{i} \int f_{i} d \mu \\
& =\sum_{i=1}^{n} a_{i} \mu\left(A_{f_{i}}\right)=\sum_{i=1}^{n} a_{i} \hat{\mu}\left(f_{i}\right) \\
& =\hat{\mu}\left(\sum_{i=1}^{n} a_{i} f_{i}\right)=\hat{\mu}(g) .
\end{aligned}
$$

It is therefore clear that if $\hat{\mu}$ is the zero function on $T$, then $\mu$ is the zero measure. Thus $\mu \rightarrow \hat{\mu}$ is one-to-one.

We complete the proof of the theorem by showing that $\mu \rightarrow \hat{\mu}$ preserves multiplication. Let $\mu$ and $\nu$ be measures on $A$, and let $f$ be in $T$. We have $(\mu * \nu)^{\wedge}(f)$ $=\mu * \nu\left(A_{f}\right)=\mu \times \nu\left(\alpha^{-1}\left(A_{f}\right)\right)$, where $\alpha: S \times S \rightarrow S$ is the multiplication map on $S$. Since the complement of $A_{f}$ is a prime ideal, $\alpha^{-1}\left(A_{f}\right)=A_{f} \times A_{f}$. Thus

$$
\mu \times \nu\left(\alpha^{-1}\left(A_{f}\right)\right)=\mu \times \nu\left(A_{f} \times A_{f}\right)=\mu\left(A_{f}\right) \nu\left(A_{f}\right)=\hat{\mu}\left(A_{f}\right) \hat{v}\left(A_{f}\right) .
$$

Therefore, $(\mu * \nu)^{\wedge}=\hat{\mu} \hat{\nu}$, and hence $\mu \rightarrow \hat{\mu}$ preserves multiplication.

We hope to invert the transform $\mu \rightarrow \hat{\mu}$ by obtaining an explicit formula for computing $\mu$ in terms of the values of $\hat{\mu}$ on $T$. Since $\mu(E)=\hat{\mu}\left(\chi_{E}\right)(E \in A)$ when we consider $\hat{\mu}$ as a linear functional on the simple functions, our approach is to express $\chi_{E}$ as a linear combination of semicharacters and use the linearity of $\hat{\mu}$. It clearly suffices to compute $\chi_{E}$ where $E$ is a fundamental set. If we expand the right-hand side of (2), we obtain

$$
\chi_{B(X, \sigma)}=\sum_{\tau \geqq \sigma}(-1)^{|\tau|-|\sigma|} \prod_{i=1}^{n} f_{i}^{\tau(i)}
$$

where $|\tau|=\sum_{i=1}^{n} \tau(i)$ counts the number of ones in the $n$-tuple $\tau$. Formula (4) motivates the following definition. 
Definition 2.2. Let $X=\left\{f_{1}, \ldots, f_{n}\right\}$ be a finite subset of $T$, let $\sigma \in T_{n}$, and let $F$ be a function on $T$. Define an operator $L$ on the triple $X, \sigma$, and $F$ by

$$
L(X, \sigma) F=\sum_{\tau \geqq \sigma}(-1)^{|\tau|-|\sigma|} F\left(\prod_{i=1}^{n} f_{i}^{\tau(i)}\right) .
$$

We let $f^{0}=1$ for any $f \in T$.

The operator $L$ provides an explicit formula for inverting the transform $\mu \rightarrow \hat{\mu}$. The value of $\mu$ on a fundamental set $B(X, \sigma)$ is $\mu(B(X, \sigma))=L(X, \sigma) \hat{\mu}$. This is the inversion formula we sought.

We conclude the section by pointing out the close relationship between our inversion formula and the classical Möbius inversion formula. (Rota's paper [7] contains the necessary background information on the Möbius and zeta functions on a finite partially ordered set, and a proof of the Möbius inversion formula [7, Proposition 2].) The above relationship is clear if we make appropriate notational adjustments. Fix the set $X=\left\{f_{1}, \ldots, f_{n}\right\}$ of semicharacters in $T$. Let the Boolean algebra $T_{n}$ be the partially ordered set under consideration. A measure $\mu$ on $A$ is considered a function on $T_{n}$ by defining

$$
\hat{\mu}(\sigma)=\hat{\mu}\left(\prod_{i=1}^{n} f_{i}^{\sigma(t)}\right) .
$$

Note that $\prod_{i=1}^{n} f_{i}^{\sigma(i)}$ is the characteristic function of the set $\bigcup_{\imath \geqq \sigma} B(X, \tau)$. Since $\mu$ is finitely additive,

$$
\hat{\mu}(\sigma)=\mu\left(\bigcup_{\tau \geqq \sigma} B(X, \tau)\right)=\sum_{\tau \geqq \sigma} \mu(\tau)=\sum_{\tau \in T_{n}} \zeta(\sigma, \tau) \mu(\tau),
$$

where $\zeta$ is the zeta function for $T_{n}$. We can solve for $\mu$ as a function on $T_{n}$ using Möbius function for $T_{n}$ (denoted throughout this paper by $m$ ). We obtain

$$
\mu(\sigma)=\sum_{\tau} m(\sigma, \tau) \hat{\mu}(\tau)=\sum_{\tau \geqq \sigma}(-1)^{|\tau|-|\sigma|} \hat{\mu}(\tau) .
$$

The second equality follows since $T_{n}$ is a Boolean algebra, in which case $m(\sigma, \tau)$ $=(-1)^{|\tau|-|\sigma|}[7$, Proposition 5].

3. Properties of the map $\mu \rightarrow \hat{\mu}$, and a Radon-Nikodym theorem. The terminology of $\S 2$ will be used throughout this section. We introduce notions such as positive definite and bounded variation for functions defined on $T$ with the aid of our inversion operator $L$ (cf. Definition 2.2).

Definition 3.1. A function $F$ on $T$ is positive definite if $L(X, \sigma) F \geqq 0$ for each finite subset $X=\left\{f_{1}, \ldots, f_{n}\right\}$ of $T$ and each $\sigma$ in $T_{n}$.

Definition 3.2. A function $F$ on $T$ is of bounded variation if $\sup \sum_{\sigma}|L(X, \sigma) F|$ $<\infty$, where the supremum is taken over all finite subsets $X$ of $T$. The norm of $F$ is the number $\sup _{X} \sum_{\sigma}|L(X, \sigma) F|$.

Definition 3.3. A function $G$ on $T$ is absolutely continuous with respect to a positive definite function $F$ on $T$ if given $\varepsilon>0$, there is a $\delta>0$ such that, for any 
finite subset $X=\left\{f_{1}, \ldots, f_{n}\right\}$ of $T$ and any subset $H$ of $T_{n}, \sum_{\sigma \in H} L(X, \sigma) F<\delta$ implies $\sum_{\sigma \in H}|L(X, \sigma) G|<\varepsilon$. If $G$ is absolutely continuous with respect to $F$, we write $G<F$.

These definitions reduce to the usual definitions of bounded variation and absolute continuity on the unit interval if we let $S=[0,1]$, with maximum multiplication, and $T=\left\{\chi_{[0, x]} \mid x \in[0,1]\right\}$, and we identify each $x \in[0,1]$ with the semicharacter $\chi_{[0, x]}$. Note that $T$ is isomorphic to $[0,1]$, with minimum multiplication. If $F$ is a function on $[0,1]$ and $X=\left\{x_{1}, \ldots, x_{n}\right\}$ is a subset of $[0,1]$ with $0 \leqq x_{1}$ $\leqq x_{2} \cdots \leqq x_{n}=1$, then $\sum_{\sigma \in T_{n}}|L(X, \sigma) F|=\left|F\left(x_{1}\right)\right|+\sum_{i=2}^{n}\left|F\left(x_{i}\right)-F\left(x_{i-1}\right)\right|$. Thus a positive definite function in the example is a nonnegative, nondecreasing function, and a function absolutely continuous with respect to $F(x)=x$ is an absolutely continuous function in the classical sense. Moreover, a function on $T$ is of bounded variation in the sense of Definition 3.2 if and only if it is of bounded variation in the classical sense. It is hoped this example justifies the above terminology.

The following theorem shows how the map $\mu \rightarrow \hat{\mu}$ relates function and measure theoretic concepts. The proof is straightforward and is omitted. Measure theoretic notions involved can be found in [3].

THEOREM 3.1. The isomorphism $\mu \rightarrow \hat{\mu}$ has the following properties.

(1) $\mu \rightarrow \hat{\mu}$ maps positive measures on $A$ to positive definite functions on $T$.

(2) $\mu \rightarrow \hat{\mu}$ maps bounded measures on $A$ to functions of bounded variation on $T$ in a norm preserving manner.

(3) $\mu \rightarrow \hat{\mu}$ preserves absolute continuity; i.e., if $\nu$ is a measure absolutely continuous with respect to the positive measure $\mu$, then $\hat{v}$ is a function absolutely continuous with respect to the positive definite function $\hat{\mu}$.

THEOREM 3.2. The algebra $M(A)$ of all bounded, finitely additive measures on $A$, with convolution multiplication, is a Banach algebra. The algebra $B V(T)$ of all functions of bounded variation on $T$, with pointwise multiplication and bounded variation norm, is also a Banach algebra. The map $\mu \rightarrow \hat{\mu}$, defined in Theorem 2.1, maps $M(A)$ isomorphically and isometrically onto $B V(T)$.

Proof. The proof follows almost trivially from Theorem 2.1 and Theorem 3.1. The convolution product of two bounded measures $\mu$ and $\nu$ in $M(A)$ is again a bounded measure; in fact $\|\mu * \nu\| \leqq\|\mu \times \nu\|=\|\mu\|\|\nu\|$. Since $M(A)$ is a Banach space, the above norm inequality shows that $M(A)$ is a Banach algebra. Combining the results of Theorem 2.1 and Theorem 3.1, we conclude that $\mu \rightarrow \hat{\mu}$ maps $M(A)$ isomorphically and isometrically onto $B V(T)$. Therefore, $B V(T)$ is a Banach algebra with pointwise operations and the bounded variation norm given it above. This completes the proof of the theorem.

We conclude this section with a discussion of the Radon-Nikodym theorem in our setting. Let $\nu$ be a bounded measure and $\mu$ be a positive measure with $\nu<\mu$. Let $w_{X}$ be a simple function on $S$ defined by

$$
w_{X}=\sum_{\sigma} \frac{\nu(B(X, \sigma))}{\mu(B(X, \sigma))} \chi_{B(X, \sigma)}
$$


for each finite subset $X$ of $T$. We adopt the convention that $\nu(B(X, \sigma)) / \mu(B(X, \sigma))=0$ whenever $\mu(B(X, \sigma))$ (and hence $\nu(B(X, \sigma)))$ is zero. A corresponding measure $\nu_{X}$ is defined by $\nu_{X}(E)=\int_{E} w_{X} d \mu$ for each set $E$ in $A$. Clearly, we have $\nu_{X}<\mu$. The collection $\left\{v_{X}\right\}$ of all such measures forms a net, with the ordering given by $X \leqq Y$ if $X \subset Y$. The following theorem shows that the net $\left\{v_{X}\right\}$ converges to $\nu$ in total variation norm.

THEOREM 3.3. Let $\nu$ and $\mu$ be two bounded measures on $A$ with $\mu$ positive and $\nu$ absolutely continuous with respect to $\mu$. Then the net $\left\{\nu_{X}\right\}$ defined above converges to $\nu$ in total variation norm, and the corresponding net $\left\{w_{X}\right\}$ of simple functions converges in $\mu$-measure.

Proof. The proof we give depends strongly on a result obtained by Darst [2]. We state Darst's result in the form most convenient for our purposes. Let $\mu$ be a positive measure in the space of all bounded, finitely additive measure on some set algebra. Then measures of the form $\nu(E)=\int_{E} w d \mu$, where $w$ is a simple function with respect to the given set algebra, are dense, in total variation norm, in the set of all measures absolutely continuous with respect to $\mu$.

Let $\varepsilon>0$. Choose a simple function $w_{1}$ such that the measure $\nu_{1}(E)=\int_{E} w_{1} d \mu$ satisfies $\left\|\nu_{1}-\nu\right\|<\varepsilon / 2$. Darst's result guarantees the existence of such a function. The simple function $w_{1}$ can be written in the form $\sum_{\sigma} a_{\sigma} \chi_{B(X, \sigma)}$ for some finite subset $X$ of $T$ and constants $a_{\sigma}$. Solving for the constants $a_{\sigma}$, we obtain

$$
\nu_{1}(B(X, \sigma))=\int_{B(X, \sigma)} w_{1} d \mu=a_{\sigma} \mu(B(X, \sigma)),
$$

and hence

$$
a_{\sigma}=\nu_{1}(B(X, \sigma)) / \mu(B(X, \sigma)) .
$$

A similar argument shows that if $Y \supset X$, so that $\{B(Y, \sigma)\}$ is a refinement of $\{B(X, \sigma)\}$, then $w_{1}$ can be written

$$
w_{1}=\sum_{\sigma} \frac{\nu_{1}(B(Y, \sigma))}{\mu(B(Y, \sigma))} \chi_{B(Y, \sigma)} .
$$

For any $Y \supset X$, we have

$$
\begin{aligned}
\left\|\nu_{1}-\nu_{Y}\right\| & =\int\left|w_{1}-w_{Y}\right| d \mu \\
& =\sum_{\sigma} \int_{B(Y, \sigma)}\left|\frac{\nu_{1}(B(Y, \sigma))}{\mu(B(Y, \sigma))}-\frac{\nu(B(Y, \sigma))}{\mu(B(Y, \sigma))}\right| d \mu \\
& =\sum_{\sigma}\left|\nu_{1}(B(Y, \sigma))-\nu(B(Y, \sigma))\right| \leqq\left\|\nu_{1}-\nu\right\|<\varepsilon / 2 .
\end{aligned}
$$

Therefore,

$$
\left\|\nu-\nu_{Y}\right\| \leqq\left\|\nu-\nu_{1}\right\|+\left\|\nu_{1}-\nu_{Y}\right\|<\varepsilon / 2+\varepsilon / 2=\varepsilon
$$

for any $Y \supset X$. Thus the net $\left\{\nu_{X}\right\}$ converges to $\nu$ in total variation norm. 
The second part of the theorem follows immediately from the first. The net $\left\{\nu_{x}\right\}$ is convergent, and is therefore a Cauchy net. Hence given $\varepsilon>0$, there is a finite subset $X$ of $T$ such that for all finite subsets $Y$ and $Z$ containing $X$,

$$
\int\left|w_{Y}-w_{Z}\right| d \mu=\left\|\nu_{Y}-\nu_{Z}\right\|<\varepsilon .
$$

It is therefore clear that

$$
\mu\left(\left\{s \in S|| w_{Y}-w_{Z} \mid \geqq \varepsilon\right\}\right)<\varepsilon .
$$

Thus the net $\left\{w_{X}\right\}$ of simple functions converges in $\mu$-measure. This completes the proof.

The net $\left\{w_{X}\right\}$ is the closest thing to a Radon-Nikodym derivative of $\nu$ with respect to $\mu$ that is possible in our setting since the measures $\nu$ and $\mu$ are only finitely additive. If $\nu$ and $\mu$ are extendable to countably additive measures on some $\sigma$ algebras over $S$ which contains $A$, then $\left\{w_{X}\right\}$ converges in $L^{\prime}(\mu)$ norm to the actual Radon-Nikodym derivative of $\nu$ with respect to $\mu$. The important feature of the above theorem is that it allows us to compute the Radon-Nikodym net $\left\{w_{X}\right\}$ directly from the functions $\hat{v}$ and $\hat{\mu}$. In fact, $w_{X}$ is given by

$$
w_{X}=\sum_{\sigma} \frac{L(X, \sigma) \hat{v}}{L(X, \sigma) \hat{\mu}} \chi_{B(X, \sigma)} .
$$

The quotient $L(X, \sigma) \hat{\nu} / L(X, \sigma) \hat{\mu}$ plays the same role as the difference quotient $(G(x)-G(y)) /(F(x)-F(y))$ does in defining the derivative $d G / d F$ for functions on $[0,1]$.

4. Functions of bounded variation on idempotent semigroups. The definitions of bounded variation, bounded variation norm, and positive definiteness given in Definitions 3.1 and 3.2 can be made for functions on any abelian idempotent semigroup $T$ with identity, even though $T$ is not given as a semigroup of semicharacters on an underlying semigroup $S$. Accordingly, we let $T$ be an abelian idempotent semigroup with identity, and we show that the set $B V(T)$ of all functions of bounded variation on $T$, with pointwise operations and bounded variation norm, is a convolution measure algebra in the sense of [8]. We then study $B V(T)$ using the structure theory for commutative convolution measure algebras developed in [8].

The definition of the operator $L(X, \sigma)$ clearly makes sense if $X$ is taken as a finite subset of an abelian idempotent semigroup $T$ with identity (cf. Definition 2.2). As in Definition 3.1, we say that a function $F$ on $T$ is of bounded variation if $\sup _{X} \sum_{\sigma}|L(X, \sigma) F|<\infty$, where the supremum is taken over all finite subsets $X$ of $T$. The norm of $F$ is the number $\|F\|=\sup _{X} \sum_{\sigma}|L(X, \sigma) F|$. We say that $F$ is positive definite if $L(X, \sigma) F \geqq 0$ for each finite subset $X$ of $T$ and each $\sigma$ as in Definition 3.2. A partial ordering is introduced into $B V(T)$ as follows: $G \leqq F$ if and only if $F-G$ is positive definite. It is easy to verify 
LEMMA 4.1. The space $B V(T)$ is a partially ordered, normed linear space.

We now proceed to show that $B V(T)$ is a convolution measure algebra. A convolution measure algebra [8, Definition 2.1] is a complex $L$-space [8, Definition 1.1] with a multiplication which makes it a Banach algebra and relates appropriately to the norm and the order. Kakutani [4] was first to consider real $L$-spaces, and Rieffel [6] first introduced complex $L$-spaces. The Banach space of all bounded, finitely additive measures on a Boolean algebra is a complex $L$-space. Thus our approach is to show that $B V(T)$ is a complex $L$-space by producing a linear isometry from $B V(T)$ onto the space of all bounded, finitely additive measures on a certain Boolean algebra. This Boolean algebra is constructed as follows. For each $x \in T$, let $P_{x}$ denote the translation operator on $B V(T)$ defined by

$$
P_{x} F(y)=F(x y) \quad(F \in B V(T), y \in T) .
$$

The following facts concerning translation operators are immediate.

LEMMA 4.2. Each translation operator on $B V(T)$ is a projection (idempotent) operator. Any pair of translation operators commute, and, in fact, $P_{x} P_{y}=P_{x y}$ for $x, y \in T$. The translation operator $P_{1}$, where 1 is the identity in $T$, is the identity operator.

Lemma 4.2 shows the linear span of the translation operators is a commutative ring with identity. We let $B$ denote the set of all projection operators contained in the linear span of the translation operators. The lattice operations of least upper bound $(\vee)$, greatest lower bound $(\wedge)$, and complement are introduced in $B$ as follows. For each pair of projection operators $P, Q \in B$, define $P \vee Q=P+Q-P Q$, and $P \wedge Q=P Q$. The complement of a projection operator $P$ is $I-P$, where $I$ is the identity operator. It is easy to see that $B$ is a Boolean algebra with respect to the above operations. This method of obtaining a Boolean algebra from a commutative ring with identity is a very standard one [1]. The Boolean algebra $B$ is the one we have been seeking. Our order preserving linear isometry $\beta$ from $B V(T)$ onto the space of all bounded, finitely additive measures on $B$ will be defined by

$$
\beta F(P)=P F(1)
$$

for each $F \in B V(T)$ and $P \in B$.

The following definitions are needed in the sequel.

Definition 4.1. Let $X=\left\{x_{1}, x_{2}, \ldots, x_{n}\right\}$ be a finite subset of $T$. For each $\sigma \in T_{n}$, the operator

$$
P(X, \sigma)=\sum_{\tau \in T_{n}} m(\sigma, \tau) P_{\tau}
$$

will be called a fundamental operator, where $m$ is the Möbius function for $T_{n}$ and $P_{\tau}$ is the translation operator $P_{y}$, where $y=\prod_{i=1}^{n} x_{i}^{\tau(t)}$. 
Definition 4.2. A set of operators $\left\{P_{i}\right\}_{i=1}^{n}$, with each $P_{i} \in B$, is called a decomposition of the identity $I$ if

(1) $P_{i} P_{j}=0$ if $i \neq j$

(2) $P_{1} \vee P_{2} \vee \cdots \vee P_{n}=\sum_{i=1}^{n} P_{i}=I$.

Definition 4.3. If $P, Q \in B$, then $Q \leqq P$ if and only if $P Q=P$. A decomposition $\left\{Q_{j}\right\}_{j=1}^{m}$ is called a refinement of the decomposition $\left\{P_{i}\right\}_{i=1}^{n}$ if each $Q_{j}$ satisfies $Q_{j} \leqq P_{i}$ for some $i(1 \leqq i \leqq n)$.

The next two lemmas demonstrate a close relationship between fundamental operators and the fundamental sets considered in $\$ 2$. In Lemma 4.3, we show that a fundamental operator is projection (idempotent) operator in $B$, and that the collection $\{P(X, \sigma)\}\left(\sigma \in T_{n}\right)$ of fundamental operators form a decomposition of the identity in the sense of Definition 4.2. In Lemma 4.4, we show that any decomposition of the identity can be refined by a decomposition of the form $\{P(X, \sigma)\}$ for some appropriate choice of $X$. The proofs we give of these lemmas, however, are quite different from the corresponding ones in $\$ 2$. Roughly speaking, the algebraic machinery of semicharacters and prime ideals is replaced by the combinatorial analysis of the Möbius and zeta functions. Another important connection between the fundamental sets and fundamental operators is the following. Since the formula $P(X, \sigma) F(1)=L(X, \sigma) F(F \in B V(T))$ holds for the operator $P(X, \sigma)$, it follows from (6) that

$$
\beta F(P(X, \sigma))=P(X, \sigma) F(1)=L(X, \sigma) F .
$$

A similar relationship between measures on $A$ and functions on $T$ was obtained in $\S 2$.

Lemma 4.3. If $X=\left\{x_{1}, \ldots, x_{n}\right\}$ is a finite subset of $T$, then each operator $P(X, \sigma) \in B$ for each $\sigma \in T_{n}$, and the set of operators $\{P(X, \sigma)\}_{\sigma \in T_{n}}$ is a decomposition of the identity.

Proof. The fact that each $P(X, \sigma) \in B$ and that $P(X, \sigma) P(X, \tau)=0$ for $\sigma \neq \tau$ follow from the factorization

The formula

$$
P(X, \sigma)=\left[\prod_{\sigma(i)=1} P_{x_{i}}\right]\left[\prod_{\sigma(i)=0}\left(I-P_{x_{i}}\right)\right]
$$

$$
P_{\sigma}=\sum_{\tau \in T_{n}} \zeta(\sigma, \tau) P(X, \tau) \quad\left(\sigma \in T_{n}\right)
$$

is verified with the aid of the Möbius inversion formula ([7], see also discussion at the end of $\S 2$ ). An application of (10) with $\sigma$ equal to the zero $n$-tuple, and hence $P_{\sigma}=I$, yields the expression $\sum_{\tau \in T_{n}} P(X, \tau)=I$. This completes the proof of the lemma.

LEMMA 4.4. Any decomposition of the identity may be refined by a decomposition of the form $\{P(X, \sigma)\}$ for some finite subset $X$ of $T$.

Proof. Let $\left\{P_{i}\right\}_{i=1}^{n}$ be a decomposition of the identity where each $P_{i}$ is a linear combination of translation operators $P_{x_{i j}}$ for $1 \leqq j \leqq n_{i}$. Set $X_{i}=\bigcup_{j=1}^{n_{i}} x_{i j}$ and 
$X=\bigcup_{i=1}^{n} X_{i}$. It can be shown that, if $X_{i} \subset X$, then there exist subsets $H_{i} \subset T_{m}$ (assuming that $X$ contains $m$ points) such that $P_{i}=\sum_{\sigma \in H_{i}} P(X, \sigma)$ for $1 \leqq i \leqq n$. Furthermore, the sets $H_{i}$ may be chosen so that $P(X, \tau) \neq 0$ for $\tau \in H_{i}$. This observation is verified by writing out $P_{i}$ as a linear combination of translation operators and then applying formula (9) to each translation operator. We claim that the sets $H_{i}$ are pairwise disjoint. For if $\tau \in H_{i} \wedge H_{j}(i \neq j)$, then $P(X, \tau) P_{i}=P(X, \tau)$ and $P(X, \tau) P_{j}=P(X, \tau)$. If we multiply the first equation by $P_{j}$, we obtain $P(X, \tau)=0$, a contradiction. Thus, $I=\sum_{i=1}^{n} P_{i}=\sum_{\sigma \in K} P(X, \sigma)$, where $K=\bigcup_{i=1}^{n} H_{i}$. Finally, observe that $\tau \in K$ if and only if $P(X, \tau) \neq 0$. Thus if $P(X, \tau) \neq 0$, then $\tau \in H_{i}$ for some $i$, and hence $P(X, \tau) P_{i}=P(X, \tau)\left(P(X, \tau) \leqq P_{i}\right)$. Therefore, the decomposition $\{P(X, \sigma)\}$ refines $\left\{P_{i}\right\}_{i=1}^{n}$, and the proof is complete.

THEOREM 4.1. There is an order preserving, linear isometry $\beta$ from the space $B V(T)$ of all functions of bounded variation on $T$ to the space $M(B)$ of all bounded, finitely additive measures on the Boolean algebra $B$. The image $\beta F$ of a function $F \in B V(T)$ is defined by $\beta F(P)=P F(1)$ for each $P \in B$.

Proof. Clearly $\beta$ is a linear map. To show that $\beta F$ is finitely additive on $B$, let $P$ and $Q$ be disjoint elements of $B$; i.e., $P \vee Q=P Q=0$. Then $P \vee Q=P+Q-P Q$ $=P+Q$, and

$$
\beta F(P \vee Q)=(P+Q) F(1)=P F(1)+Q F(1)=\beta F(P)+\beta F(Q) .
$$

Now $\beta F$ is bounded if sup $\sum_{i=1}^{n}\left|\beta F\left(P_{i}\right)\right|<\infty$, where the supremum is taken over all decompositions $\left\{P_{i}\right\}_{i=1}^{n}$ of the identity. Lemma 4.4 shows that this supremum may be taken over all decompositions $\{P(X, \sigma)\}$, where $X$ is a finite subset of $T$. Therefore,

$$
\begin{aligned}
\|\beta F\| & =\sup _{X} \sum_{\sigma}|\beta F(P(X, \sigma))|=\sup _{X} \sum_{\sigma}|P(X, \sigma) F(1)| \\
& =\sup _{X} \sum_{\sigma}|L(X, \sigma) F|=\|F\|<\infty
\end{aligned}
$$

where $F \in B V(T)$. The third equality follows from (7). We have shown that $\beta$ is an isometry.

To prove that $\beta$ is onto, let $\lambda$ be a bounded finitely additive measure on $B$. Define a function $F$ on $T$ by $F(x)=\lambda\left(P_{x}\right)$ for each $x \in T$. We claim that $\beta F=\lambda$. It suffices to show that $\beta F$ and $\lambda$ agree on the fundamental operators $P(X, \sigma)$. The equality $\beta F(P(X, \sigma))=\lambda(P(X, \sigma))$ can be obtained by writing out $P(X, \sigma) F(1)$, using (9), and the Möbius inversion formula. Thus $\beta$ is onto. Finally, (7) shows that $F$ is positive definite if and only if $\beta F$ is a positive measure on $B$. Hence $\beta$ is order preserving. The proof of the theorem is complete.

THEOREM 4.2. The algebra $B V(T)$ of all functions of bounded variation on $T$ is a commutative convolution measure algebra, with pointwise addition and multiplication of functions, and bounded variation norm. 
Proof. Theorem 4.1 shows that $B V(T)$ is a complex $L$-space. We need to show that $B V(T)$ is a Banach algebra, and that the three conditions listed in Definition 2.1 of [8] hold. The essential ingredient in our proof is the formula

$$
P(X, \sigma)(F G)=\sum_{\tau_{1} \wedge \tau_{2}=\sigma}\left[P\left(X, \tau_{1}\right) F\right]\left[P\left(X, \tau_{2}\right) G\right],
$$

where $X=\left\{x_{1}, \ldots, x_{n}\right\}$ is a finite subset of $T, \sigma \in T_{n}$, and $F, G \in B V(T)$. The proof of (10) involves formula (9), the Möbius inversion formula, and the equality

$$
\zeta\left(\tau, \tau_{1}\right) \zeta\left(\tau, \tau_{2}\right)=\zeta\left(\tau, \tau_{1} \wedge \tau_{2}\right) \quad\left(\tau, \tau_{1}, \tau_{2} \in T_{n}\right),
$$

where $\zeta$ is the zeta function for $T_{n}$.

The first two conditions of Definition 2.1 of [8] and the fact that $B V(T)$ is a Banach algebra follow from (10). For suppose $F, G \in B V(T)$. Using (7), and evaluating the operators in (10) at the identity yields

$$
L(X, \sigma)(F G)=\sum_{\tau_{1} \wedge \tau_{2}=\sigma}\left[L\left(X, \tau_{1}\right) F\right]\left[L\left(X, \tau_{2}\right) G\right] .
$$

The norm inequality $\|F G\| \leqq\|F\|\|G\|$ follows from (11), with equality whenever $F$ and $G$ are positive definite. Thus $B V(T)$ is a Banach algebra, and the first condition of Definition 2.1 of [8] holds. The second condition, namely that the product of two positive definite functions is positive definite, follows trivially from (11).

The third condition is a little more difficult to verify. Let $F, G$, and $H$ be positive definite functions on $T$ with $0 \leqq H \leqq F G$. Since the map $\beta$ defined in Theorem 4.1 preserves order, we have $0 \leqq \beta H \leqq \beta(F G)$. Clearly, $\beta H$ is absolutely continuous with respect to $\beta(F G)$. Recall that Darst's Radon-Nikodym theorem ([2], see also Theorem 3.3) says the following: if $\nu$ and $\mu$ are bounded, finitely additive measures on a Boolean algebra, and $\nu$ is absolutely continuous with respect to the positive measure $\mu$, then given $\varepsilon>0$, there is a partition $\left\{E_{i}\right\}_{i=1}^{n}$ such that $\left\|\nu-\left.\sum_{i=1}^{n} a_{i} \mu\right|_{E_{i}}\right\|<\varepsilon$. The measure $\left.\mu\right|_{E_{i}}$ is defined by $\left.\mu\right|_{E_{i}}(E)=\mu\left(E \cap E_{i}\right)$ for each set $E$ in the given Boolean algebra. We now fix $\varepsilon>0$. According to the above remarks, we can choose a finite subset $X=\left\{x_{1}, \ldots, x_{n}\right\}$ of $T$ and constants $a_{\sigma}\left(\sigma \in T_{n}\right)$ such that

$$
\left\|\beta H-\left.\sum_{\sigma} a_{\sigma} \beta(F G)\right|_{P(X, \sigma)}\right\|<\varepsilon .
$$

The constants $a_{\sigma}$ may be chosen such that $0 \leqq a_{\sigma} \leqq 1$ since $\beta H \leqq \beta(F G)$. Note that $\left.\beta F\right|_{P}=\beta(P F)$ since

$$
\left.\beta F\right|_{P}(Q)=\beta F(P Q)=P Q F(1)=Q(P F)(1)=\beta(P F)(Q)
$$

for any $P$ and $Q$ in our Boolean algebra $B$ of operators. The above inequality can then be written

$$
\left\|\beta H-\sum_{\sigma} a_{\sigma} \beta P(X, \sigma)(F G)\right\|<\varepsilon .
$$

Since $\beta$ is an isometry, we have

$$
\left\|H-\sum_{\sigma} a_{\sigma} P(X, \sigma)(F G)\right\|<\varepsilon .
$$


An application of formula (10) shows that

$$
\begin{aligned}
\sum_{\sigma} a_{\sigma} P(X, \sigma)(F G) & =\sum_{\sigma} a_{\sigma} \sum_{\tau_{1} \wedge \tau_{2}=\sigma} P\left(X, \tau_{1}\right) F P\left(X, \tau_{2}\right) G \\
& =\sum_{\tau_{1}, \tau_{2}} a_{\tau_{1} \tau_{2}} P\left(X, \tau_{1}\right) F P\left(X, \tau_{2}\right) G
\end{aligned}
$$

where $a_{\tau_{1} \tau_{2}}=a_{\sigma}$ whenever $\tau_{1} \wedge \tau_{2}=\sigma$. We therefore have

$$
\left\|H-\sum_{\tau_{1}, \tau_{2}} a_{\tau_{1} \tau_{2}} P\left(X, \tau_{1}\right) F P\left(X, \tau_{2}\right) G\right\|<\varepsilon .
$$

The fact that $\sum_{\tau_{1}} P\left(X, \tau_{1}\right) F=F, \sum_{\tau_{2}} P\left(X, \tau_{2}\right) G=G$, and the fact that $0 \leqq a_{\tau_{1} \tau_{2}} \leqq 1$ for $\tau_{1}, \tau_{2}$ in $T_{n}$, complete the verification of the third condition. The proof of the theorem is complete.

We now investigate the structure of the algebra $B V(T)$. The point evaluation map $h_{x}$, defined by $h_{x}(F)=F(x)$ for each $F$ in $B V(T)$, is easily seen to be a complex homomorphism of $B V(T)$ for each $x$ in $T$. The point evaluation homomorphisms separate points in $B V(T)$, and hence $B V(T)$ is semisimple. Accordingly, there is a compact topological semigroup $S$ and an embedding $F \rightarrow F_{S}$ of $B V(T)$ into $M(S)$ (the measure algebra on $S$ ) such that each complex homomorphism of $B V(T)$ has the form $h_{f}(F)=\int_{S} f d F_{S}$ for some continuous semicharacter $f$ in $\hat{S}$ [8]. The space $\hat{S}$ of all continuous semicharacters on $S$, with the weak* topology, can be considered the maximal ideal space of $B V(T)$. We let $f_{x}$ be the semicharacter in $\hat{S}$ which corresponds to the complex homomorphism $h_{x}$; in this manner, each point in $T$ is identified with a semicharacter in $\hat{S}$. We prove in the theorem below that the correspondence $x \rightarrow f_{x}$ is a semigroup isomorphism from $T$ into the semigroup of semicharacters $S$ with pointwise multiplication. We further let $M$ denote the $L$ subalgebra of $M(S)$ which is the image of the map $F \rightarrow F_{S}$. An $L$-subalgebra of $M(S)$ is an $L$-subspace [8, Definition 1.1] which is closed under convolution multiplication. Since $\hat{S}$ is the maximal ideal space of $B V(T)$, it can obviously be considered the maximal ideal space of $M$. Thus the map $\mu \rightarrow \hat{\mu}$, defined by $\hat{\mu}(f)$ $=\int_{S} f d \mu(\mu \in M, f \in \hat{S})$, is the Gelfand transform. If the transform $\hat{\mu}$ is restricted to the set $T$ by letting $\hat{\mu}(x)=\int_{S} f_{x} d \mu$ for each $x$ in $T$, then it becomes a function on $T$. We denote by $\left.\mu \rightarrow \hat{\mu}\right|_{T}$ the map which carries a measure $\mu$ in $M$ to its Gelfand transform restricted to $T$. The equalities

$$
F(x)=h_{x}(F)=\int_{S} f_{x} d F_{S}=\hat{F}_{S}(x) \quad(F \in B V(T), x \in T)
$$

show that the map $\left.\mu \rightarrow \hat{\mu}\right|_{T}$ is the inverse of the map $F \rightarrow F_{S}$. In particular, the function $\left.\hat{\mu}\right|_{T}$ is in $B V(T)$ for each $\mu$ in $M$. We formalize our remarks in the following theorem.

THEOREM 4.3. Let $T$ be an abelian idempotent semigroup with identity. Then there exists a compact, abelian topological semigroup $S$, an L-subalgebra $M$ of $M(S)$, and a semigroup isomorphism $x \rightarrow f_{x}$ of $T$ into $\hat{S}$ such that: 
(1) Every complex homomorphism $h$ of $M$ has the form $h(\mu)=\int_{S} f d \mu=\hat{\mu}(f)$ for some $f$ in $\hat{S}$.

(2) If we consider $T \subset \hat{S}$ via the embedding $x \rightarrow f_{x}$, then the map $\left.\mu \rightarrow \hat{\mu}\right|_{T}$ is an isomorphism and order preserving isometry of $M$ onto $B V(T)$.

Proof. In view of our discussion preceding the theorem, we need only show that $x \rightarrow f_{x}$ is a semigroup isomorphism. This is done by producing a semigroup isomorphism $x \rightarrow g_{x}$ of $T$ into $\hat{S}$ and then showing that $f_{x}=g_{x}$ for each $x$ in $T$. Our proof depends strongly on results obtained by Taylor in [8]. We let $R$ denote the ring of bounded operators from $B V(T)$ to $B V(T)$ which leave $L$-subspaces invariant [8, Definition 1.2]. It was shown in Lemma 3.1 of [8] that $Q \in R$, then $Q$ is an algebraic homomorphism if and only if there is an $f \in \hat{S} \cup\{0\}$ such that $(Q F)_{S}(V)$ $=\int_{V} f d F_{S}$ for each $F$ in $B V(T)$ and each Borel subset $V$ of $S$. The algebraic homomorphisms in $R$, under composition, form a semigroup of operators. The above correspondence $Q \rightarrow f$ is a semigroup isomorphism, a fact which follows from Theorem 1.1 of [8] and $\S 3$ of [8].

The translation operators $P_{x}$ are elements of the ring $R$. This fact becomes clear if we consider functions in $B V(T)$ as measures via the order preserving isometry $\beta$ of Theorem 4.1. The equality $\beta\left(P_{x} F\right)=\left.\beta F\right|_{P_{x}}$ (the measure $\beta F$ restricted to $P_{x}$ ) shows that each $P_{x}$ is a bounded linear operator from $B V(T)$ into $B V(T)$ which leaves $L$-subspaces invariant. Thus each translation operator is in $R$. Lemma 4.1 shows that $P_{x y}=P_{x} P_{y}$ for any $x$ and $y$ in $T$. It follows that the map $x \rightarrow P_{x}$ is a semigroup isomorphism, and that each translation operator $P_{x}$ is an algebraic homomorphism. The remarks of the previous paragraph now assure the existence of a semicharacter $g_{x}$ in $\hat{S}$ which corresponds to the algebraic homomorphism $P_{x}$ for each $x$ in $T\left(g_{x} \neq 0\right.$ since $\left.P_{x} \neq 0\right)$. These remarks also show that the map $P_{x} \rightarrow g_{x}$ is an isomorphism. Composition of the maps $x \rightarrow P_{x}$ and $P_{x} \rightarrow g_{x}$ yields a semigroup isomorphism $x \rightarrow g_{x}$ of $T$ into $\hat{S}$.

We now show that $f_{x}=g_{x}$ for each $x$ in $T$. Combining results of the first two paragraphs, we obtain

$$
\begin{aligned}
\int_{S} f_{x} d F_{S} & =h_{x}(F)=F(x) \\
& =P_{x} F(1)=\left(P_{x} F\right)_{S}(S)=\int_{S} g_{x} d F_{S}
\end{aligned}
$$

for each $F$ in $B V(T)$. The equality $g_{x}=f_{x}$ now follows from the fact that the measures $F_{S}$ separate continuous functions on $S$ (cf. [8]).

We conclude that the map $x \rightarrow f_{x}$ is a semigroup isomorphism. This completes the proof.

The map $x \rightarrow f_{x}$ embeds $T$ in $\hat{S}$ as an idempotent subsemigroup which separates points in $B V(T)$. The semigroup $\hat{S}$, however, need not be idempotent. The counterexample we give is a modification of one given in [5]. Let $T^{\prime}$ be the countable 
product of the two semigroups $\{0,1\}$, under multiplication. Observe that $T^{\prime}$ is a compact, idempotent semigroup. The measure algebra $M\left(T^{\prime}\right)$, under convolution, is a semisimple convolution measure algebra. We showed in [5] that the structure semigroup of $M\left(T^{\prime}\right)$ is not idempotent. We now let $T=\left\{f_{x}\right\}_{x \in T^{\prime}}$ be an idempotent semigroup of semicharacters on $T^{\prime}$, where $f_{x}$ is the characteristic function of the set $\left\{y \in T^{\prime} \mid y \geqq x\right\}$. Let $A$ be the algebra of subsets of $T^{\prime}$ generated by kernels of semicharacters in $T$ as in $\S 2$. The map which takes $M\left(T^{\prime}\right)$ to $M(A)$ by restricting a measure in $M\left(T^{\prime}\right)$ to the set algebra $A$ is one-to-one.

Thus $M\left(T^{\prime}\right)$ can be considered an $L$-subalgebra of $M(A)$, and hence of $B V(T)$, via the isomorphism-isometry of $\S 2$, Theorem 2.1. Since the structure semigroup of $M\left(T^{\prime}\right)$ is not idempotent, it follows that the structure semigroup $S$ of $B V(T)$ (and hence the semigroup $\hat{S}$ ) is not idempotent [5, Theorem 1].

\section{BIBLIOGRAPHY}

1. G. Birkhoff, Lattice theory, 3rd ed., Amer. Math. Soc. Colloq. Publ., vol. 25, Amer. Math. Soc., Providence, R. I., 1967. MR 37 \#2638.

2. R. B. Darst, A decomposition of finitely additive set functions, J. Reine Angew. Math. 210 (1962), 31-37. MR $25 \# 1257$.

3. N. Dunford and J. T. Schwartz, Linear operators. I: General theory, Pure and Appl. Math., vol. 7, Interscience, New York, 1958. MR 22 \#8302.

4. S. Kakutani, Concrete representation of abstract $(L)$-spaces and the mean ergodic theorem, Ann. of Math. (2) 42 (1941), 523-537. MR 2, 318.

5. S. E. Newman, Measure algebras on idempotent semigroups, Pacific J. Math. 31 (1969), 161-169.

6. M. A. Rieffel, A characterization of commutative group algebras and measure algebras, Trans. Amer. Math. Soc. 116 (1965), 32-65. MR 33 \#6300.

7. G.-C. Rota, On the foundations of combinatorial theory. I. Theory of Möbius functions, Z. Wahrscheinlichkeitstheorie und Verw. Gebiete 2 (1964), 340-368. MR 30 \#4688.

8. J. L. Taylor, The structure of convolution measure algebras, Trans. Amer. Math. Soc. 119 (1965), 150-166. MR 32 \#2932.

Department of Mathematics, University of Missouri, St. Louis, Missouri 63122 